\title{
ANÁLISIS COMPARATIVO DE LA MORTALIDAD MATERNA EN CHILE, CUBA Y ESTADOS UNIDOS DE NORTEAMÉRICA
}

\author{
Drs. Enrique Donoso S., Enrique Oyarzún E. \\ Departamento de Obstetricia y Ginecología. Facultad de Medicina. Pontificia Universidad Católica de Chile
}

\section{RESUMEN}

En 2000, la mortalidad materna en Chile, Cuba y EEUU, fue de 18,7/100.000, 40,4/100.000 y 9,8/ 100.000 nacidos vivos respectivamente, diferencias estadísticamente significativas. La tasa de mortalidad materna por causas directas en Chile fue significativamente menor que en Cuba y mayor que en EEUU; mientras que la por causas indirectas no presentó diferencias significativas con Cuba y fue significativamente mayor que en EEUU. La tasa de muerte materna por aborto en Chile, Cuba y EEUU fue de 4,6/ $100.000,2,8 / 100.000$ y $0,3 / 100.000$ nacidos vivos respectivamente, diferencias explicadas por el aborto legalizado. Chile fue el único de los tres países que redujo la mortalidad materna en más de $50 \%$ entre 1990-2000.

\section{PALABRAS CLAVES: Mortalidad materna, estadísticas vitales}

\section{SUMMARY}

In 2000, the maternal mortality in Chile, Cuba and USA was 18.7, 40.4 and 9.8/100.000 live births respectively, differences that were significant. The chilean direct maternal mortality rate was significantly lower than Cuba and higher than USA; while indirect maternal mortality rate was not statistically different than Cuba and significantly higher than USA. Abortion maternal mortality rate in Chile, Cuba and USA was 4.6, 2.8 and $0.3 / 100.000$ live births respectively, differences explained by legal abortion. Chile was the only of those countries who reduced the maternal mortality by more than $50 \%$ in $1990-2000$ period.

\section{KEY WORDS: Maternal mortality, vital statistics}

\section{INTRODUCCIÓN}

La tasa de mortalidad materna es un indicador que refleja el grado de desarrollo sanitario, cultural y económico de un país. Es en este indicador donde se establecen las brechas más amplias entre los países según su nivel de desarrollo. Los países desarrollados tienen tasas de mortalidad materna promedio de 21/100.000 nacidos vivos, mientras que los en vías de desarrollo y en los menos desarrollados presentan tasas de $440 \mathrm{y}$
$1.000 / 100.000$ nacidos vivos respectivamente (1). En la Región de las Américas, las enormes diferencias entre los países determinan que el rango de mortalidad materna oscile entre 4 y 523/ 100.000 nacidos vivos, dado por Canadá y Haití respectivamente $(2,3)$. Los múltiples factores que inciden en la reducción de la mortalidad materna hacen que Chile, Argentina y Costa Rica fuesen los únicos países latinoamericanos que pudieron mostrar reducciones sostenidas del indicador en la década de los noventa $(4,5)$. 
El objetivo de este estudio es analizar la mortalidad materna de tres países americanos con claras diferencias sociales, culturales, económicas y de sus sistemas de salud, como son los casos de Chile, Cuba y Estados Unidos de Norteamérica.

\section{MATERIAL Y MÉTODO}

Se efectúa un análisis descriptivo y comparativo de la mortalidad materna de Chile, Cuba y Estados Unidos de Norteamérica (EEUU), correspondiente al año 2000. El número de muertes maternas, de nacidos vivos (NV) y las causas de muerte, se extraen de las bases de datos de estadísticas vitales de los países analizados (6-9). Se define muerte materna al fallecimiento de la mujer durante el embarazo, parto o puerperio, debido a causas propias (directas) o agravadas (indirectas) por el proceso reproductivo, con exclusión de las causas accidentales e incidentales. Las causas de muerte materna se presentan agrupadas según códigos de la décima revisión de la Clasificación Internacional de Enfermedades, Traumatismos y Causas de Defunción (CIE-10). El análisis comparativo entre los países se presenta según causas de muertes proporcionada por las bases de datos de Cuba y EEUU, a las cuales se adecua la de Chile. La comparación entre proporciones se efectuó mediante la prueba de Chi cuadrado, con un nivel de significación estadística de $p<0,05$.

\section{RESULTADOS}

En 2000, la tasa de mortalidad materna en Chile fue de 18,7/100.000 NV, en Cuba de 40,4/ 100.000 NV y en EEUU de 9,8/100.000 NV, diferencias estadísticamente significativas entre ellas (Tabla I). La tasa global de mortalidad materna por causas directas fue significativamente menor en Chile que en Cuba y mayor que en EEUU; mientras que en las indirectas no hubo diferencia significativa con Cuba y fue significativamente mayor que en EEUU (Tablas II y III). Las 3 principales causas de muerte materna en Chile fueron las relacionadas con el aborto $(4,6 / 100.000 \mathrm{NV})$, la hipertensión arterial $(4,6 / 100.000 \mathrm{NV})$ y las enfermedades pregestacionales (2,7/100.000 NV); en Cuba fueron las relacionadas con las complicaciones derivadas del trabajo de parto y parto $(7,0 /$ 100.000 NV), la hipertensión arterial (5,6/100.000 $\mathrm{NV}$ ) y las complicaciones derivadas del puerperio $(4,9 / 100.000 \mathrm{NV})$, y en EEUU las relacionadas con la hipertensión arterial $(1,8 / 100.000 \mathrm{NV})$, la embolia obstétrica $(1,4 / 100.000 \mathrm{NV})$ y las causas indirectas $(1,4 / 100.000 \mathrm{NV})$ (Tablas II y III).
Tabla I

NACIDOS VIVOS Y MORTALIDAD MATERNA (X 100.000 NV) EN CHILE, CUBA Y EEUU, 2000

\begin{tabular}{lccc}
\hline País & $\begin{array}{c}\text { Nacidos } \\
\text { vivos }\end{array}$ & $\begin{array}{c}\text { Muertes } \\
\text { maternas }\end{array}$ & $\begin{array}{c}\text { Mortalidad } \\
\text { materna }\end{array}$ \\
\hline Chile & 261.993 & 49 & $18,7^{\mathrm{a}}$ \\
Cuba & 143.528 & 58 & $40,4^{\mathrm{a}, \mathrm{b}}$ \\
EEUU & 4.058 .814 & 396 & 9,8 \\
\hline
\end{tabular}

aDiferencias significativas con EEUU.

${ }^{b}$ Diferencia significativa con Chile.

\section{DISCUSIÓN}

Los resultados de este estudio muestran que la mortalidad materna en EEUU fue significativamente menor a la de Chile y Cuba, lo que es consistente con el nivel de desarrollo económico y sanitario de esos países; en el año analizado, el Producto Interno Bruto de EEUU, Chile y Cuba fue respectivamente de 34.637, 9.623 y 2.712 dólares internacionales, mientras que el gasto en salud per cápita fue respectivamente de 4.499, 697 y 186 dólares internacionales (10).

Las complicaciones derivadas del aborto son una de las causas más importantes de muerte materna en los países Latinoamericanos y del Caribe (11). En 2000, la mortalidad materna por aborto en Chile fue de 4,6/100.000 NV, en Cuba de 2,8/100.000 NV y en EEUU de 0,3/100.000 NV. La menor tasa de mortalidad materna por aborto en Cuba y EEUU se explica por la legalización y amplia liberalización del aborto inducido (12), los que son realizados institucionalmente por profesionales médicos, mientras que en Chile el aborto es ilegal en cualquier circunstancia (13) y efectuado habitualmente en forma clandestina por agentes no médicos. En Cuba durante 1996, se realizaron 209.900 abortos legales, con tasas de 77,7 abortos/1000 mujeres de 15-44 años y 58,6 abortos/100 embarazos, mientras que en EEUU en el mismo año, hubo 1.365 .700 abortos legales, con tasas de 22,9 abortos/1000 mujeres de 15-44 años y 25,9 abortos/100 embarazos (14). En Chile se desconoce la cifra real de abortos inducidos, estimándose una cifra de 160.000 abortos sobre la base de cálculos derivados de las hospitalizaciones por abortos complicados, con tasas de 50 abortos/1000 mujeres de 15-44 años y 35,3 abortos/100 embarazos (15). Evitar la muerte materna por embarazo no deseado es uno de los fundamentos básicos del uso de la anticoncepción y del 
Tabla II

TASAS DE MORTALIDAD MATERNA (X 100. 000 NV) POR GRUPOS DE CAUSAS DE MUERTE EN CUBA Y CHILE, 2000

\begin{tabular}{|c|c|c|c|c|}
\hline \multirow[t]{2}{*}{ Causas de muerte materna (CIE-10) } & \multicolumn{2}{|c|}{ Cuba } & \multicolumn{2}{|c|}{ Chile } \\
\hline & $n$ & Tasa & $n$ & Tasa \\
\hline Directas* & 47 & 32,8 & 40 & 15,3 \\
\hline Embarazo ectópico (O00) & 5 & 3,5 & 1 & 0,4 \\
\hline Aborto $(001-008)$ & 4 & 2,8 & 12 & 4,6 \\
\hline Complicaciones relacionadas con el puerperio (O85-O87, O89-092) & 7 & 4,9 & 2 & 0,8 \\
\hline Embolia obstétrica (O88) & 3 & 2,1 & 3 & 1,1 \\
\hline Complicaciones del trabajo de parto y parto (O60, O61, O66, O68-071, 073-O75) & 10 & 7,0 & - & - \\
\hline Anomalía de la dinámica del parto (O62) & 7 & 4,9 & 2 & 0,8 \\
\hline Placenta previa y abruptio placentae (O43-O45) & 3 & 2,1 & - & - \\
\hline Otras hemorragias $(\mathrm{O} 20,046,067,072)$ & - & - & 3 & 1,1 \\
\hline Trastornos hipertensivos (011-O16) & 8 & 5,6 & 12 & 4,6 \\
\hline Otras complicaciones & - & - & 5 & 1,9 \\
\hline Indirectas ${ }^{\star \star}$ & 11 & 7,7 & 9 & 3,4 \\
\hline $\begin{array}{l}\text { Enfermedades infecciosas y parasitarias, clasificables en otra parte pero que complican } \\
\text { el embarazo, el parto y el puerperio (098) }\end{array}$ & 2 & 1,4 & 1 & 0,4 \\
\hline $\begin{array}{l}\text { Otras enfermedades maternas clasificables en otra parte pero que complican el } \\
\text { embarazo, el parto y el puerperio (O99) }\end{array}$ & 2 & 1,4 & 7 & 2,7 \\
\hline Otras causas indirectas & 7 & 4,9 & 1 & 0,4 \\
\hline
\end{tabular}

${ }^{*}$ Diferencia significativa.

${ }^{* *}$ Diferencia no significativa.

aborto inducido legal, pese a lo cual, en EEUU entre 1972 y 1998, hubo 342 muertes maternas por aborto inducido legal (16).

Es motivo de controversia la relación entre los niveles de uso de anticonceptivos y aborto inducido. El pensamiento habitual es que el aumento de la anticoncepción disminuirá consecuentemente el aborto inducido. Estudios poblacionales muestran que en países donde la fecundidad presenta un descenso constante, el mayor uso de anticonceptivos disminuye la incidencia de aborto inducido, situación que correspondería a Chile; mientras que el aumento paralelo de la anticoncepción y del aborto inducido ocurre en aquellos países donde

Tabla III

TASAS DE MORTALIDAD MATERNA (X 100. 000 NV) POR GRUPOS DE CAUSAS DE MUERTE EN EEUU Y CHILE, 2000

\begin{tabular}{|c|c|c|c|c|}
\hline \multirow[t]{2}{*}{ Causas de muerte materna (CIE-10) } & \multicolumn{2}{|c|}{ EEUU } & \multicolumn{2}{|c|}{ Chile } \\
\hline & $n$ & Tasa & $n$ & Tasa \\
\hline Directas* $^{*}$ & 341 & 8,4 & 40 & 15,3 \\
\hline Embarazo ectópico (O00) & 25 & 0,6 & 1 & 0,4 \\
\hline Aborto $(001-008)$ & 12 & 0,3 & 12 & 4,6 \\
\hline Complicaciones relacionadas con el puerperio (O85-092) & 35 & 0,9 & 2 & 0,8 \\
\hline Embolia obstétrica (O88) & 58 & 1,4 & 3 & 1,1 \\
\hline Hemorragias periparto $(\mathrm{O} 44-\mathrm{O} 46, \mathrm{O} 67, \mathrm{O} 72)$ & 30 & 0,7 & 3 & 1,1 \\
\hline Trastornos hipertensivos (011, 013-016) & 72 & 1,8 & 12 & 4,6 \\
\hline Muerte por causa no especificada (O95) & 4 & 0,1 & - & - \\
\hline Otras complicaciones & 105 & 2,6 & 7 & 2,7 \\
\hline Indirectas* & 55 & 1,4 & 9 & 3,4 \\
\hline $\begin{array}{l}\text { Enfermedades maternas parasitarias e infecciosas (O98) y otras enfermedades (O99), } \\
\text { clasificables en otra parte pero que complican el embarazo, el parto y el puerperio }\end{array}$ & 55 & 1,4 & 9 & 3,4 \\
\hline
\end{tabular}

*Diferencia significativa 
la fecundidad disminuye agudamente y la anticoncepción no satisface la creciente necesidad de la población de regular la fecundidad, como ocurriría en Cuba y EEUU (17). En Chile la anticoncepción se inicia en la década de los 60 , observándose desde entonces, una asociación entre la reducción de las hospitalizaciones derivadas de las complicaciones del aborto y las muertes maternas por esa causa (18). Actualmente Chile se encuentra en proceso de transición demográfica, es así que la tasa global de fecundidad en 1990 fue de 2,6/ hijos por mujer y en el 2000 de 2,1 (6), reducción equivalente a $19,2 \%$ en una década, por lo que el aumento de la cobertura anticoncepcional podría reducir aún más las muertes por embarazo no deseado. Las cifras oficiales de prevalencia de usuarias de anticonceptivos en el país es de $56 \%$, una de las más bajas en Sudamérica (19).

La mortalidad materna relacionada con la hipertensión arterial es la primera causa de muerte en EEUU ( $1,8 / 100.000 \mathrm{NV})$ y Chile $(4,6 / 100.000$ $\mathrm{NV})$, siendo en Cuba una de las principales $(5,6 /$ 100.000 NV). La reducción de la mortalidad por esa causa es dependiente de la cobertura y calidad del control prenatal, como también del control de la fecundidad en los grupos de alto riesgo hipertensivo como son las mujeres mayores de 39 años y las adolescentes. Chile, desde ese punto de vista, tiene un riesgo mayor por el significativo aumento de los nacimientos en esos grupos etarios $(20,21,22)$, con tasas de fecundidad de 13,5 nacimientos/1000 mujeres de 40-44 años, en comparación a EEUU con 7,9 y Cuba con 3,0 (6, 7, 9); mientras que en adolescentes la tasa de fecundidad en Chile es 64,1 nacimientos/1000 mujeres de 15-19 años, en Cuba de 52,3 y en EEUU de 48,5 (6, 7, 9). Pese a eso, la mortalidad materna en adolescentes chilenas es menor que la de los otros países analizados; la tasa para el período 1990 - 1999 fue en Chile de 7,6/100.000 NV, en Cuba de 20,4 en 1999 y en EEUU de 8,6 para el período 1991-1999 $(22,23,24)$. Es destacable la baja tasa de mortalidad materna en adolescentes chilenas comparadas con las de Cuba y EEUU, países que tienen al embarazo adolescente como causal de aborto inducido legal $(12,25)$.

La tasa de mortalidad materna en Chile por enfermedades pregestacionales (código 099) fue mayor que la de Cuba y EEUU. Es posible que en esos países el aborto terapéutico influya en la reducción de sus tasas. En Chile, la muerte materna por enfermedades pregestacionales aporta un promedio de 7 fallecimientos por año, sin evidenciarse una tendencia a la reducción y es la prime- ra causa de muerte materna en mujeres mayores de 39 años $(4,20,21)$.

Llama la atención que las principales causas de muerte materna en Cuba se relacionen con complicaciones derivadas de la atención del parto y del puerperio, país con 530 médicos $/ 100.000$ habitantes y $99 \%$ de atención profesional del parto, en comparación a Chile y EEUU con 110 y 279 médicos/100.000 habitantes respectivamente, donde esas causas tienen una importancia menor y con cobertura similar a Cuba en la atención profesional de la embarazada (10).

En 1990, la mortalidad materna en EEUU fue de 10,0/100.000 NV (26), en Chile de 39,9 (4) y en Cuba de 31,6 (23), con un porcentaje de cambio para el año 2000 de $+26,9 \%$ en Cuba, $-2,0 \%$ en EEUU y $-53,1 \%$ en Chile. Eso significa que de los 3 países, solamente Chile cumplió con el Plan de Acción Regional para la Reducción de la Mortalidad Materna en las Américas (27), en el cual los países de la Región se comprometieron a reducirla en 50\% para el período 1990-2000 (28). EEUU no pudo reducir la mortalidad materna según los objetivos programados para 2000 , que era una tasa de 3,3/100.000 NV y mantiene el mismo objetivo para 2010 (29), mientras que Chile planifica reducir la tasa a 12/100.000 NV para el mismo año (30); no disponemos de esa información para Cuba.

La mortalidad materna sigue siendo un tema prioritario para todas las naciones, especialmente para los países en vías de desarrollo, de ahí que surjan nuevos compromisos internacionales, el último de ellos refrendado por los gobernantes de 149 países que se comprometieron a reducir la mortalidad materna en $75 \%$ en 2015 (31).

Es posible concluir que en EEUU y Cuba el control de la mortalidad materna es fuertemente dependiente del aborto inducido legal, mientras que Chile mantiene una sostenida reducción del indicador analizado con pleno respeto de la vida humana en gestación $(13,32,33)$.

\section{BIBLIOGRAFÍA}

1. World Health Organization, United Nations Children's Fund, United Nations Population Fund. Maternal mortality in 1995: Estimates developed by WHO, UNICEF, UNFPA. World Health Organization, Geneva, Reproductive Health and Research, 2001.

2. Canadian Perinatal Health Report, 2000. Arbuckle T, Dzakpasu S, Liu S, Rouleau J, Rusen ID, Turner L, Wu Wen S. Disponible en: www.hc-sc.gc.ca/hpb/lcdc/ brch/reprod.html. Acceso el 20 de enero de 2004.

3. Ministère de la Santé Publique et de la Population. 
Mortalité maternelle. Chapitre 12. En: Enquête Mortalité, Morbidité et Utilization des Services. EMMUS-III. Haïti, 2000. Disponible en: http:// 63.99.209.162/publications/emmus_3. Acceso el 20 de enero de 2004.

4. Donoso E, Poblete J, Villarroel L. Mortalidad materna. Chile, 1990-1996. Rev Chil Obstet Ginecol 1998; 63(4): 290-7.

5. AbouZahr C, Wardlaw T. Maternal mortality at the end of a decage: Sign of progress? Bull World Health Org 2001, 79(6): 561-8.

6. Instituto Nacional de Estadísticas. Anuario de Estadísticas Vitales. Chile, 2000.

7. Anuario Estadístico de Salud. Cuba, 2000. Disponible en: www.sld.cu/servicios/estadisticas/. Acceso el 20 de enero de 2004.

8. Miniño AM, Arias E, Kochanek KD, Murphy SL, Smith BL. Deaths: Final Data for 2000. National Vital Statistics Reports, Vol. 50, № 15. Hyattsville, Maryland: National Center for Health Statistics, 2002.

9. Martin JA, Hamilton BE, Ventura SJ, Menacker F, Park MM. Birth: Final Data for 2000. National Vital Statistics Reports, Vol, 50, № 5. Hyattsville, Maryland: National Center for Health Statistics, 2002.

10. World Health Organization. Indicadores básicos sanitarios y económicos de los Estados miembros. Disponible en: www.who.int/country/en/index.html. Acceso el 20 de enero de 2004.

11. Langer A. El embarazo no deseado: impacto sobre la salud y la sociedad en América Latina y el Caribe. Rev Panam Salud Pública 2002; 11(3): 192-204.

12. David HP, de Weiss S. El aborto en las Américas. En: López J, Yunes J, Solís JA, Omran AR, (eds). SALUD REPRODUCTIVA EN LAS AMERICAS. Capítulo 13, pp 353-86. Organización Panamericana de la Salud. OPS/OMS, 1992.

13. Código Sanitario. Libro V. Artículo 119. Ley $\mathrm{N}^{\circ}$ 18.826 del 15 de septiembre, 1989.

14. Henshaw SK, Sing S, Haas T. The incidence of abortion worldwide. International Family Planning Perspectives 1999; 25(Suppl): S30-7.

15. Sing S, Wulf D. Estimated levels of abortion in six Latin American countries. International Family Planning Perspectives 1994; 20(1): 4-3.

16. Elam-Evans LD, Strauss LT, Herndon J, Parker WY, Whitehead S, Berg,CJ. Abortion Surveillance United State,1999. En: Surveillance Summaries, November 29, 2002. MMWR; 51(№ SS-9): 1-32.

17. Marston C, Cleland J. Relationships between contraception and abortion: A review of the evidence. International Family Planning Perspectives 2003; 29(1): 6-13.

18. Viel B, Campos W. La experiencia chilena de mortalidad infantil y materna, 1940-1985. Perspectivas Internacionales en Planificación Familiar 1987; (Número Especial): 24-8.
19. UNICEF. Health statistical data. Country data. Contraceptive use. Disponible en: http://www.unicef.org/ statis/atoz.html\#c. Acceso el 20 de enero de 2004.

20. Donoso E, Becker J, Villarroel L. Evolución de la natalidad y del riesgo reproductivo en mujeres de 40 o más años en la década de los 90 . Rev Chil Obstet Ginecol 2002, 67(2): 139-42.

21. Donoso E, Villarroel L. Edad materna avanzada y riesgo reproductivo. Rev Méd Chile 2003; 131(1): 559.

22. Donoso E, Becker J, Villarroel L. Natalidad y riesgo reproductivo en adolescentes de Chile, 1990-1999. Rev Panam Salud Pública 2003; 14(1): 3-8.

23. Cabezas E. Mortalidad materna y perinatal en adolescentes. Rev Cubana Obstet Ginecol 2002; 28(1): 5-10.

24. Chang J, Elam-Evans LD, Berg Cj, Herndon J, Flowers L, Seed KA. Pregnancy-related mortality surveillance - United States, 1991-1999. In: Surveillance Summaries. MMWR 2003; 52(SS-2): 1-8.

25. Spitz AM, Velebil P, Koonin LM y cols. Pregnancy, abortion and birth rates among US adolescents 1980, 1985 and 1990. JAMA 1996; (13)275: 989-94.

26. Berg CJ, Atrash HK, Koonin LM, Tucker M. Pregnancy-related mortality in the United States, 1987-1990. Obstet Gynecol 1996; 88(2): 161-7.

27. Donoso E. Plan de Acción Regional para la Reducción de la Mortalidad Materna en las Américas: Resultados de Chile. Rev Chil Obstet Ginecol 2003(1): 13-6.

28. OPS, OMS. XXIII Conferencia Sanitaria Panamericana. Washington, DC, Septiembre de 1990. Plan de Acción Regional para la Reducción de la Mortalidad Materna en las Américas. Documento CSP23, R17. Disponible en: http://165.158.1.110/spanish/db// FULLTEXT/ftcsp_23.htm. Acceso el 20 de enero de 2004.

29. US Department of Health and Human Services. Healthy People 2010. $2^{\text {nd }}$ ed. With understanding and improving health and objectives for improving health (2 vols.). Washington, DC: US Department of Health and Human Services, 2000. Disponible en: www.cdc.gov/nchs. Acceso el 20 de enero de 2004.

30. Ministerio de Salud de Chile. Objetivos sanitarios y modelo de salud para la década 2000-2010. Documento oficial, enero de 2002. Disponible en: www.minsal.cl. Acceso el 20 de enero de 2004.

31. Naciones Unidas. La Declaración del Milenio. Resolución A/RES/55/2. New York, 2000. Disponible en: http://www.un.org/spanish/millenniumgoals/ ares552.htm. Acceso el 20 de enero de 2004.

32. Valenzuela C. Etica científica del aborto terapéutico. Rev Méd Chile 2003; 131(5): 562-8.

33. Besio M. Análisis ético sobre las interrupciones del embarazo. Ars Médica 2002, 4(6): 83-93. 